Discussion: Along with metal-framed decontamination tents, there also are inflatable tents. The inflatable decontamination units can be pitched in 5-7 minutes by an electrical air compressor. Metal-framed tents are easy to carry, as the frame, metal legs, and tent cloth are stored in carrying bags. By pitching the decontamination tent on a smooth ground in front of the hospital entrance, decontamination of patients can be completed before they reach the hospital. Another decontamination unit is in place next to the emergency department.

Conclusion: Terrorist attacks that result in mass casualties demonstrate the importance of CBRN preparedness. A decontamination unit and an appropriate CBRN preparedness plan should be constituted into the basic treatment system.

Prehosp Disaster Med 2011;26(Suppl. 1):s122-s123 doi:10.1017/S1049023X11004067

(P1-75) Medical Measurement against Disaster - From the Viewpoint of "Disaster Medicine Compendium" Y. Haraguchi

Surgery, Tokyo, Japan

The world must be resilient against major disasters, whether they are caused by natural hazards or human-related mechanisms. Nowadays, outbreaks of emerging infectious diseases, such as new strains of influenza, are a world wide problem. Special consideration is necessary against chemical, biological, radiological, or nuclear (CBRN) hazards.

Results: The hardware/mechanical preparedness, measurements of the severe influence in mental/psychological aspects, official public system, incident command system, public education system, and the needs of vulnerable populations should be established prior to a disaster. It is also important to prepare the necessary medical resources. The technical skills needed for triage, emergency care, and appropriate transportation should be trained for.

Discussion and Conclusion: Measures should be prepared both from the macroscopic viewpoint as well as microscopic approach. A "Disaster Medicine Compendium" consisting of 20 volumes with more than 5,000 pages, was developed in 2005. Currently, a new version is being prepared.

Prehosp Disaster Med 2011;26(Suppl. 1):s123 doi:10.1017/S1049023X11004079

(P1-76) Descriptive Study of Poisoning Victims Patients at a Tertiary Medical Center in Northern Thailand P.C. Inboriboon, ${ }^{1}$ P. Narongchai ${ }^{2}$

1. Department of Medicine and Emergency Medicine, San Diego, United States of America

2. Toxicology, Chiang Mai, Thailand

Background: Poisoning is a public health problem that effects all ages, genders, and ethnicities, but certain populations are at increased risk. Unintentional poisoning disproportionately affects lower- and middle-income countries. The epidemiological pattern of poisoning can differ significantly within countries. In Thailand, national poisoning data is collected by voluntary reporting to a poison control center in the capital, Bangkok.
Limited poisonings are reported from Chiang Mai, a province in Northern Thailand, though it has the highest suicide rate in the country.

Objective: The aim of this study is to describe poisoning epidemiology, examine differences between intentional and unintentional poisonings and, to develop a multivariate predictive model of intentional poisonings in Northern Thailand.

Methods: A total of 550 poisoning admissions were identified at Chiang Mai's University Hospital from 01 January 2005 to 31 December 2005. Demographic data were collected retrospectively by manual chart review. Chi-square and t-tests were used to examine differences in poisoning by intent. A stepwise logistic regression analysis was performed to develop a predictive model of intentional poisonings. Factors significantly associated with intentional poisoning were used in a logistic progression model to identify predictors of intentional poisoning.

Results: Younger age was significantly associated with intentional poisoning. There was no demonstrated difference in gender and poisoning intent. Predictors of intentional poisonings were use of chemical agents, high-risk medications (analgesics and psychiatric), unemployment, and younger age.

Conclusions: Interventions should focus on factors that put young adults and the unemployed at greater risk for intentional poisonings. Further interventions are also needed to limit access to potentially lethal doses of chemical agents and high risk medications.

Prehosp Disaster Med 2011;26(Suppl. 1):s123

doi:10.1017/S1049023X11004080

\section{(P1-77) Helping Children Cope with Ongoing Stress Y. Arbeli}

District Health Office, Ashkelon, Israel

Stress is a major health risk factor. The origin of the stress or stressful situations might come from internal and/or external causes. In this presentation, two groups of Israeli children who are living under stressful conditions that are affecting their health, their daily functioning, and their learning abilities will be presented. The first group is a group of children living in a town that has experienced terrorist activities for many years. The other group is children from families that have to leave their permanent home due to the Israeli Parliamentary and governmental decision to withdraw from the Gaza Strip. This plan included forced relocation of approximately 8,000 civilians from their communities to temporary sites elsewhere in Israel, and the dismantling of their homes. Using the classical epidemiological triad model of host-agent-environment, the hazard dynamic and its outcomes will be presented. Activities to help the children cope with the stressful situations also will be presented. The objective of this presentation is to describe exposure to risk factors and responses from public health nurses that are aimed at ameliorating the associated negative heath effects.

Prehosp Disaster Med 2011;26(Suppl. 1):s123

doi:10.1017/S1049023X11004092 